\title{
Controlled Optimal Black Start Procedures in Smart Grids for Service Restoration in Presence of Electrical Storage Systems
}

\author{
Alessandro Giuseppi ${ }^{\dagger}$, Antonio Pietrabissa, Francesco Liberati, Alessandro Di Giorgio \\ Department of Computer, Control, and Management Engineering Antonio Ruberti (DIAG) \\ Sapienza University of Rome \\ Via Ariosto 25, 00185, Rome, Italy \\ $\dagger$ Corresponding Author: giuseppi@diag.uniroma1.it
}

\begin{abstract}
This paper presents an optimisation problem to determine the optimal reclosure order of remotely operable switches deployed in a smart grid consisting in a distribution network equipped with one or more Energy Storage Systems (ESS). The proposed solution integrates nonlinear real and reactive power flow equations, by reconducting them to a set of conic constraints, together with several network operator requirements, such as network radiality and ampacity limits. A numerical simulation validates the approach and concludes the work.
\end{abstract}

Index Terms-Service Restoration, Controlled Black Start, Power Network Resiliency, Mixed Integer Programming

\section{LIST OF MAIN SYMBOLS}

$\mathcal{A} \quad$ Adjacency matrix of the distribution network's graph

$\mathcal{V}, \mathcal{V}^{c} \quad$ Sets of: all the network nodes and reconnected nodes

$\mathcal{V}^{E S S}$ Set of the nodes equipped with a ESS,

$\theta_{i} \quad$ Voltage phase at the $i$-th node

$a_{i j} \quad$ Boolean control variable representing the closure of a breaker on line $i j$

$C_{i}^{E S S}$ Capacity of the storage at the $i$-th node

$h \quad$ TIme step

$P_{i}^{D G} \quad$ Active power of the distributed generator of the $i$-th node

$P_{i}^{\text {load }}$ Total active power of the $i$-th node

$Q_{i}^{s t} \quad$ Short term prediction of the reactive power of the $i$-th node

$Q_{i} \quad$ Total reactive power of the $i$-th node

$Q_{i}^{D G} \quad$ Reactive power related to the distributed generator of the $i$-th node

$Q_{i}^{\text {load }}$ Reactive power demand of the $i$-th node

$S O C_{i}^{E S S}$ State of Charge (percentage) of the storage at the $i$-th node

$T \quad$ Sampling time

$V_{i} \quad$ Voltage magnitude at the $i$-th node

$v_{i}^{c} \quad$ Boolean variable representing the reconnection of node $i$

This work is partially supported by the SAPIENZA - ATENEO 2017 "PROMETEO - Protezione di reti elettriche di potenza da attacchi ciberfisici mediante strategie di controllo" project, no. RM11715C7EFAF857.

\section{INTRODUCTION}

During the recent years, a significant amount of research and investments has been spent to improve the security of power systems, which transitioned from purely passive networks into an evolved Cyber Physisical System [1] in which sophisticated controller are able to provide advanced services, such as demand-side management and frequency regulation, exploiting new active devices such as Energy Storage Systems (ESSs) and Smart Home Controllers. In fact, in modern micro grids, the quantity of controllable loads and distributed generators is significantly increasing, also thanks to the degree of freedom brought by electro-mobility, and ESSs are being utilised to attain better service performances (e.g., minimize power losses, improve frequency regulation and enhance system stability [2], [3], resiliency [4]) and security [5] and their number is expected to increase significantly as distributed generation becomes even more pervasive [6] and electric vehicles become predominat [7]. This work investigates the possibility of utilising mediumsized ESSs at network distribution level, to allow the islanded operation of a smart grid when the connection with the transmission has been lost after a service disruption. The scenario in which the proposed controller will operate is the one of a complete distribution network black start [8], in the sense that it is required to restore the service provision to a portion of the distribution network after a black out and the consequent opening of all the network breakers and switched due to the automatic network protection systems. The goal of the controller is then to determine an optimal reclosing strategy for the remotely controllable network breakers that it supervises, so that the ESSs and distributed generators can sustain the network imbalance in an islanded mode (i.e., in the absence of connection with the transmission network). Regarding recent black start studies available in the literature, we note that several works, such as [9]-[11], 
are based on Mixed Integer Linear Programming to capture operational constraints and operate the system with a logic similar to the one considered in this work in both a single optimisation or receding horizon fashion. Most of the works available in the literature, as the present paper, assume the availability of a communication network to remotely operate the actuators and gather state information, while on the contrary, the authors in [12] proposed a communication-free solution. Ideally, when a short-term forecast of the incoming faults or attacks happening on the transmission network is available, a preventive controlled system separation (i.e., controlled islanded operation before a service interruption) can be actuated to avoid black outs [4], [13], but, nevertheless, a solution for black start, as the proposed controller, shall be paired with such solutions in order to be able to restore the service provision even in case of failure of the procedure.

The present work extends and generalises the results from the previous study of the authors [14], that dealt with the same black start procedure, in the following directions, that represent the major contributions of the work:

- The proposed controller integrates real and reactive power flows, meaning that the assumption of having a lossless network is removed.

- The problem is now modeled in a generalised framework derived from the one proposed in [4] in which the network topology is not assumed to be meshed but is arbitrary, meaning that the proposed controller is provided with a set of constraints to allow the operation of the network while also being compliant with typical network operator requirements, such as radiality and expected survival time of the islands.

- The controller is now able to manage systems with multiple ESSs, as it will be shown to be able to operate several different islands whose number depend on the available ESSs.

Regarding the control logic and the statement of our pbolem, we considered the following operator requirements to define the set of constraints proposed in the following:

- The network shall be operated in a radial way at all times, with the additional requirement that one radial island should span from each of the available ESSs to both minimise power losses and to prevent having a power exchange between two ESSs.

- Remotely operable breakers that have been closed shall not be re-opened, in order to avoid excessive equipment degradation. This implies also that a node for which the service was restored should not have a further service interruption, meaning that the SAIFI and SAIDI indicators are not further lowered [15].

- The control shall be computed in a reasonable amount of time, in the order of a few minutes, so that the service restoration can start immediately. This is attained thanks to the conification of the power flow equations and the limited, or local, scope of the portion of network controlled. The proposed controller has been designed to overview a medium sized portion of the network under the supervision of a regional control centre, as in larger scale systems it is more convenient to instance multiple copies of the controller after a regional network slicing.

- In order to avoid dangerous transient behaviours, a limited amount of contemporary breaker reclosures has to be allowed, as in [14], and the system sampling time shall be adequately large (in the order of several seconds to one minute).

- The optimal reconnection sequence shall be determined in such a way that the service is restored to the most loads possible, while also taking into account the expected survival time of the system.

The objective of the controller becomes then to find an optimal reconnection strategy of all the network nodes in such a way that the operative requirements of the list above are satisfied at all times, wile also optimising network losses and the survival time of the system.

The rest of the paper is organised as follows: Section II details the proposed controller, describing its objective function and the constraints that model the underlying network; Section III states the optimisation problem, summarising the discussion of the previous section; Section IV presents a numerical simulation to validate the approach. Finally, Section V draws the conclusions and highlights future works.

\section{Proposed Control System}

\section{A. Objective Function}

The discussed control objectives are captured by the following objective function, which will be minimised by the proposed controller:

$$
\begin{aligned}
& F(\mathcal{A}(h))= \\
& \sum_{h=0}^{h=t_{f}} \sum_{i=1}^{|\mathcal{V}|}\left(-\alpha v_{i}^{c}(h) P_{i}^{\text {load }}(h)-\beta S O C_{i}^{E S S}(h)+\gamma P_{i}(h)\right),
\end{aligned}
$$

where $\mathcal{A}(h)$ is the adjacency matrix of the distribution network's graph at time $h$ (as defined in Section II-B), $t_{f}$ represents the survival time requested for the islanded operation of the network and $\alpha, \beta, \gamma$ are relative weights that prioritize the three considered objectives. Minimizing the function (1) implies that our controller aims at providing as much power as possible to the network customers (first term, that will be shown to be a sum of the reconnected network nodes weighted by their loads), while also doing a trade-off with the energy reserves contained in the ESSs (second term) and minimizing power losses (third term, as it will be clear with the power flow introduction). As it will be discussed, the third objective assures also that the conification procedure $[16]^{1}$ that will be employed in the following holds. Note that in (1) it was assumed that the expected survival time is known in advance to the network operator. In cases in

\footnotetext{
${ }^{1}$ As explained in section II-D3, conification is a coordinate transformation procedure that allows the integration of nonlinear power flow equations in the optimisation problem by defying a conic proxy of the original constraints, with no effect on the solution quality.
} 
which this assumption is not reasonable, various strategies can be proposed:

- In a scenario in which the operator desires to reconnect all the nodes of the network, it is sufficient to pick $t_{f}$ equal to the number of nodes not equipped with an ESS and impose a reconnection at each time step, as it will be discussed in the following.

- From the previous point, it follows that the operator may set a fixed maximum number of nodes to be reconnected, and let the controller chose which node to reconnect and in which order.

- By imposing $t_{f}$ to be equal to an arbitrary maximum number of reconnected nodes, an additional term could be added to (1) in order to capture a trade-off between the reconnection and the expected survival time of the system. Such term should be outside the summations in (1) and act as a final cost associated to the network topology at time $t_{f}$. Given the power profile of the ESSs at time $t_{f}$, a reasonable estimation of the survival time of the network is given by assuming a steady state evolution and no fluctuations in the exogenous signals (e.g., load profiles). Introducing the auxiliary variable $\tau$ and the following set of constraints

$$
\tau<\frac{C_{i}^{E S S}}{100} \frac{S O C_{i}^{E S S}\left(t_{f}\right)}{P_{i}^{E S S}\left(t_{f}\right)} \quad \forall i \in \mathcal{V}^{E S S},
$$

it is possible to obtain the desired trade-off by maximising $\tau$, as its maximisation forces it to assume the value of the shortest survival time predicted among all the ESS (i.e., the minimum value of the right hand of (2)).

In the following section, the model of the considered network will be presented.

\section{B. System Model}

Let us model the distribution network as a graph $\mathcal{G}=$ $\{\mathcal{V}, \mathcal{A}(h)\}$, where $\mathcal{V}$ represents the set of network buses and, as already introduced, $\mathcal{A}(h)$ is the adjacency matrix at time $h$. Its Boolean elements $a(H)_{i j}=a(h)_{j i}$ are equal to 1 if, at the considered time $h$, the breaker on the link between buses $i, j$ is closed, and 0 otherwise. By the properties of the adjacency matrix, it follows that the following constraint is required

$$
a_{i j}(h)=a_{j i}(h)
$$

Furthermore, to model the fact that closed breakers shall remain in that state for all instants following their reclosure time $h$, we impose:

$$
a_{i j}(h) \leq a_{i j}(h+1) .
$$

\section{System Dynamics}

The ESSs are the dynamical elements of the network, and their state consists in the amount of energy they can provide to sustain the network. The of charge ESS $i \in \mathcal{V}^{E S S}$ at time $h, S O C_{i}^{E S S}$, is characterized by the following constrained dynamics:

$$
\begin{aligned}
& S O C_{i}^{E S S}(h+1)=S O C_{i}^{E S S}(h)-T P_{i}^{E S S}(h) \frac{100}{C_{i}^{E S S}} \\
& \text { subject to : } \\
& P_{i}^{E S S, \text { min }} \leq P_{i}^{E S S}(h) \leq P_{i}^{E S S, \text { max }} \\
& S O C_{i}^{E S S, \text { min }} \leq S O C_{i}^{E S S}(h) \leq S O C_{i}^{E S S, \text { max }},
\end{aligned}
$$

in which the parameters $P_{i}^{E S S, \min }, P_{i}^{E S S, \max }$ and $S O C_{i}^{E S S, \min }, S O C_{i}^{E S S, \max } \quad$ represent the minimum and maximum graded, or use-case specific, values for the ESS power $P_{i}^{E S S}$ and its state of charge. Note that the state of the distribution network at time $h$ is constituted also by its current topology captured by the matrix $\mathcal{A}(h)$, whose elements are the control variables that represent the breakers closure $a_{i j}(h)$.

\section{Control Constraints and Logic}

As already introduced, the logic of the controller revolves around the necessity of determining an optimal reconnection order for the network buses, so that the operational requirements identified in Section i are satisfied.

1) Network Radiality: In order to impose the radiality of the network during the entire procedure and in the consequent islanded operation, it is sufficient to impose the following constraint on the number of closed breakers at time $h$ :

$$
\sum_{\left\{i j: a_{i j}=1, j>i\right\}} a_{i j}(h) \leq\left|\mathcal{V}^{c}(h)\right|-\left|\mathcal{V}^{E S S}\right|
$$

Where $|\cdot|$ represent the cardinality operator, and the set $\mathcal{V}^{c}(h)$ contains the nodes for which the service has been restored at time $h$ (including the nodes equipped with an ESS). Constraints of the form of (6) are a common solution in network reconfiguration problems but are typically unfit for active networks, in which distributed generators and controllable ESS are present, as they may allow the operation of a nonradial network due to the formation of an autonomous island, as shown in [17]. In purely passive networks, the constraints such as (6) guarantee the radiality of the network thanks to the presence of an additional set of constraints related to the feasibility of the power flow, that implies that it is not possible for a portion of the network to operate as a non-radial island since there would be no controllable device able to feed it with power.

In our scenario, even if the considered network is a smart grid with an high penetration of distributed sources and ESS, the ESS are the devices responsible for both feeding the network and for controlling its voltage and frequency values, meaning that a separate island with no ESS cannot appear in a feasible power flow.

For these reasons, the constraint (6) in combination with the constraints of section II-D3 will assure that the network will be divided into a number of trees equal to the number 
of ESSs at any time $h$.

2) Service continuity and power quality: In order to assure proper service provision and avoid to decrease further the SAIFI/SAIDI indicators, we impose to our controller not to disconnect a load bus after it has been connected to an ESS and its power provision was restored. In terms of sets, this means

$$
\mathcal{V}_{i}^{c}(h) \subseteq \mathcal{V}_{i}^{c}(h+1) \quad \forall h .
$$

From (7) it follows that:

$$
\left|\mathcal{V}^{c}(h)\right| \leq\left|\mathcal{V}^{c}(h+1)\right| .
$$

Let the Boolean variable $v_{i}^{c}(h)$ represent the connection of node $i$ to the active portion of the network at time $h$, meaning that $v_{i}^{c}(h)=1 \Longleftrightarrow i \in \mathcal{V}^{c}(h)$, Imposing the following set of constraints

$$
v_{i}^{c}(h+1) \geq v_{i}^{c}(h), \quad \forall h
$$

one imposes to the controller not to re-interrupt the service to a node $i$.

Since the controlled variables are $a_{i j}(h)$, in order allow the controller to evaluate whether a node $i$ is connected to an ESS (i.e., the value of $v_{i}^{c}(h)$ ), the controller has to be provided with a set of constraints that directly link the network topology $\mathcal{A}(h)$ with the variables $v_{i}^{c}(h)$. This can be easily done by imposing

$$
v_{i}^{c}(h) \geq \frac{1}{|\mathcal{V}|} \sum_{j \in\{1, \ldots|\mathcal{V}|\}} a_{i j}(h) .
$$

It is trivial to verify that if $a_{i j}(h)=1$ for at least one value of $j$ (i.e., there exist a closed breaker on a link that is incident to the node $i$ ) $v_{i}^{c}(h)$ assumes the value of 1 . For the feasibility of the power flow, $a_{i j}(h)=1$ implies that there is power passing thought the line that connects the nodes $i$ and $j$, which means, for radiality of the network, that the node $i$ has been connected to a tree fed by an ESS.

Furthermore, controlling the increase of the cardinality of the set $\mathcal{V}^{c}(h)$ between two generic time instants $h$ and $h+1$, it is possible to force the controller to allow a maximum number of contemporary breaker reclosures. A reasonable choice for our case study, in the light of the operator requirements considered in section $\mathrm{I}$, is imposing a constraint of the form

$$
\begin{aligned}
& \left|\mathcal{V}^{c}(h+1)\right|-\left|\mathcal{V}^{c}(h)\right|= \\
& =\sum_{j=\{1, \ldots,|\mathcal{V}|\}}\left(v_{i}^{c}(h+1)-v_{i}^{c}(h)\right) \leq 1
\end{aligned}
$$

meaning that at most a single node is added to the set $\mathcal{V}^{c}$ at every time step. Ideally, one could allow the number of reclosures to be equal to the number of ESSs, but in order to prevent that multiple nodes are reconnected to the same ESS at the same time, and hence prevent dangerous transient behaviour and consequent power quality degradation, a fictional flow and its related conservation constraints have to be added to the control logic, as proposed in [16]. Such approach would lead to a faster reconnection of the islands, but would significantly increase the problem complexity and may delay the start of the reclosing procedure and the service restoration.

Once the evaluation of the variables $v_{i}^{c}(h)$ is made available to the controller, it is now possible to define the power flow of the network.

3) Network Power Flow: Before introducing the real and reactive power flows from node $i$ to node $j$ at time $h, P_{i j}(h)$ and $Q_{i j}(h)$, we note that due to the energy conservation law all the nodes in the network have to satisfy two power balance equations:

$$
\begin{aligned}
P_{i}(h)= & v_{i}^{c}(h)\left(P_{i}^{D G}(h)-P_{i}^{\text {load }}(h)\right)+P_{i}^{E S S}(h)= \\
& =\sum_{j} P_{i j}(h) \\
Q_{i}(h)= & v_{i}^{c}(h)\left(Q_{i}^{D G}(h)-Q_{i}^{\text {load }}(h)\right)+Q_{i}^{E S S}(h)= \\
= & \sum_{j} Q_{i j}(h)
\end{aligned}
$$

In which the terms relative to the ESS can assume nonzero values only if $i \in \mathcal{V}^{E S S}$. The presence of the variable $v_{i}^{c}(h)$ before the difference between the predicted values of power generation and demand "enables" the node from the point of view of the power flow, as it allows the right hand of the equations to assume a value different from zero (note that, by definition, if a node $i$ is equipped with a storage $\left.v_{i}^{c}(h)=1\right)$.

Denoting with $G_{i j}$ and $B_{i j}$ the line series conductance and susceptance, the real and the reactive power flows are captured by the following set of algebraic equations [18]:

$$
\begin{aligned}
P_{i j}(h)= & a_{i j}(h)\left[-G_{i j} V_{i}(h) V_{j}(h) \cos \left(\theta_{i j}(h)\right)+\right. \\
& \left.-B_{i j} V_{i}(h) V_{j}(h) \sin \left(\theta_{i j}(h)\right)+G_{i j} V_{i}^{2}(h)\right], \\
Q_{i j}(h)= & a_{i j}(h)\left[+B_{i j} V_{i}(h) V_{j}(h) \cos \left(\theta_{i j}(h)\right)+\right. \\
& \left.-G_{i j} V_{i}(h) V_{j}(h) \sin \left(\theta_{i j}(h)\right)-B_{i j} V_{i}^{2}(h)\right],
\end{aligned}
$$

where $\theta_{i j}(h)=\theta_{i}(h)-\theta_{j}(h)$ is the voltage angle difference between the two nodes and $V_{i}(h), V_{j}(h)$ are their voltage values. Note that in (14) and (15), as customary in distribution network operations [18], the shunt elements are neglected.

Following the same steps as in [4] and following the procedure originally proposed in [16], the power flow equations can be transformed into a set of quadratic constrains. This procedure will not influence the optimality of the found solution since, as fully detailed in [16], the conified problem shares the same solution with the starting nonlinear problem.

The transformation utilizes the following set of auxiliary variables: $u_{i}(h)$ for each $i \in \mathcal{V}$ and $R_{i j}(h), T_{i j}(h)$ for each line defined as:

$$
\begin{aligned}
& u_{i}(h) \leftarrow V_{i}(h)^{2} / \sqrt{2} \\
& R_{i j}(h) \leftarrow V_{i}(h) V_{j}(h) \cos \theta_{i j} \\
& T_{i j}(h) \leftarrow V_{i}(h) V_{j}(h) \sin \theta_{i j} .
\end{aligned}
$$




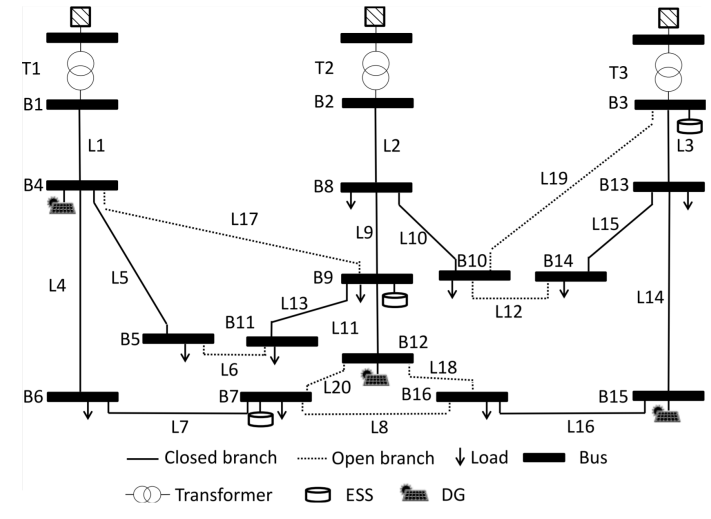

Fig. 1. Test distribution network (adapted from [20])

Under this set of substitutions, the power flow equations (14), (15) take the following form:

$$
\begin{aligned}
P_{i j}(h)= & a_{i j}(h)\left[-G_{i j} R_{i j}(h)+\right. \\
& \left.\left.B_{i j} T_{i j}(h)+G_{i j} \sqrt{(} 2\right) u_{i}(h)\right] \\
Q_{i j}(h)= & a_{i j}(h)\left[B_{i j} R_{i j}(h)+\right. \\
& \left.\left.G_{i j} T_{i j}(h)-B_{i j} \sqrt{(} 2\right) u_{i}(h)\right]
\end{aligned}
$$

As shown in [16], [19], with the addition of a new set of auxiliary variables $u_{i}^{i j}(h)$ for all nodes $i$ and lines $i j$, (17) and (18) can be reduced to a set of linear equality and conic inequality constraints. The new variables have to satisfy the following constraints in order to impose the relations $u_{i}^{i j}(h)=u_{i}(h)$ if $a_{i j}(h)=1$ and $u_{i}^{i j}(h)=0$ if $a_{i j}(h)=0$ :

$$
\begin{aligned}
& 0 \leq u_{i}^{i j}(h) \leq \frac{\left(V_{i}^{\max }\right)^{2}}{\sqrt{2}} a_{i j}(h) \\
& 0 \leq u_{i}(h)-u_{i}^{i j}(h) \leq \frac{\left(V_{i}^{\max }\right)^{2}}{\sqrt{2}}\left(1-a_{i j}(h)\right) \\
& 2 u_{i}^{i j}(h) u_{j}^{j i}(h) \geq R_{i j}(h)^{2}+T_{i j}(h)^{2}, R_{i j}(h) \geq 0 .
\end{aligned}
$$

It is trivial to verify that the constraints (19) imply the two sought relations, and, as detailed in [16], [19], as long as the third constraint is active the conified power flow shares the same solution with the original nonlinear one. The activation to the equality of the third constraint in (19) can be imposed by minimizing $R_{i j}$ [16] and this is attained by our controller thanks to the presence of the third term in the objective function (1), which represents the minimization of power losses. The power flow equations hence become [4]:

$$
\begin{gathered}
\left.P_{i j}(h)=-G_{i j} R_{i j}(h)+B_{i j} T_{i j}(h)+G_{i j} \sqrt{(} 2\right) u_{i}(h) \\
\left.Q_{i j}(h)=B_{i j} R_{i j}(h)+G_{i j} T_{i j}(h)-B_{i j} \sqrt{(} 2\right) u_{i}(h)
\end{gathered}
$$

To conclude, we mention that the variables $V_{i}(h)$ and $\theta_{i}(h)$ can be freely set on the nodes that are equipped with an ESS, within adequate operative bounds, and the original variables of the system can trivially be reconstructed from the inversion of auxiliary variables substitutions.

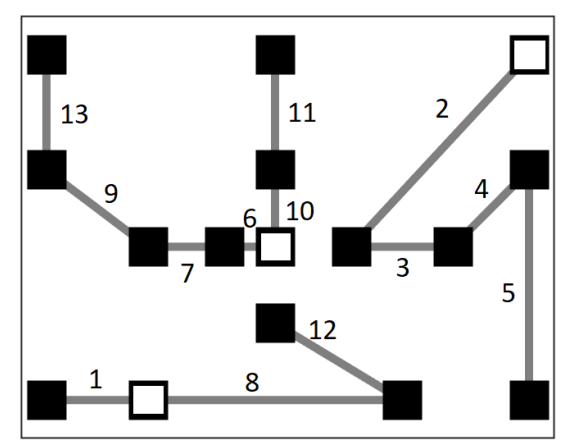

Fig. 2. Optimal breaker reclosure order. Nodes equipped with an ESS are whited.

4) Physical Limitations of the System: To assure physical feasibility and secure operation of the network, voltage and current ampacity limits have to be imposed for all nodes, lines and times:

$$
\begin{aligned}
& V_{i}^{\min } \leq V_{i}(h) \leq V_{i}^{\max }, \\
& I_{i j}(h)^{2} \leq I_{i j}^{\max 2} .
\end{aligned}
$$

Note that all other physical variables are also bounded by box constraints determined by the grading of the network equipment and are omitted for brevity.

\section{Problem Statement}

The following problem is defined:

\section{Problem 1 - Optimal Controlled Black Start}

Find $\operatorname{argmin}\{(1)\}$

subject to

(3)-(6),

(9)-(11),

(16), (19)- (22).

\section{Simulations}

The network considered for the simulation is the one depicted in Figure 1 and already investigated in [4]. It consists of 16 buses connected by a total of 20 lines. Three identical ESSs 0.5 MW / 0.5 MVA / 0.5 MWh are placed respectively on buses $3,7,9$ and three nodes are assumed to produce a net amount of power thanks to the presence of photo-voltaic panels. The loads have a mean value of $50 \mathrm{~kW}$, representing a situation in which the operator concluded a load shedding analysis to determine a quantity of power to provide critical services and infrastructures in the area, while distributed generators produce an amount of power of approximately 70 $\mathrm{kW}$.

All the lines in the considered network are assumed to be equipped with a remotely controllable breaker, making the considered network, while limited in scale, representative of larger scenarios in which the number of operable switches per HV/MV substation is lower (for both operative and economic factors). In this test we imposed to the controller to reconnect 
exactly one node at each time instant, with a 60 seconds sampling time. The weights $\alpha, \beta, \gamma$ were all set to 1 for simplicity, and the storages were assumed to start with a state of charge level between 60 and $70 \%$, losing about $4 \%$ of their energy at the end of the procedure.

The simulation was run on Julia 0.7 [21] utilising the Jump modelling language [22] and the solver Gurobi [23]. The solving time was between 3 and 5 minutes in our testing, on a ultra-low voltage $4.0 \mathrm{GHz}$ intel i7.

Figure 2 reports the optimal breaker reclosure order and the final network topology. We note that the procedure starts by reconnecting node 6 , on which there was one of the highest loads, and then it builds a tree starting from node 3 . Due to the structure of (1), renewable nodes are connected with a lower priority, with the tendency of placing them at the end of the three branches to reduce power losses. We mention that the nodes in proximity of the primary substations had a minimal quantity of load, so it is not surprising that their reconnection happens at the very last steps.

\section{CONCLUSIONS AND Future WORKS}

This paper formulated an optimisation problem for distribution network black start in presence of distributed energy sources and Energy Storage Systems (ESSs). Thanks to a conification procedure, it was possible to include in the optimisation both real and reactive power flows, together with several operative constraints to capture a series of network operator requirements, spacing from service continuity to network radiality. The proposed controller solves the resulting optimisation problem and a numerical simulation was reported to validate the approach. Future works is related to improving the scalability of the algorithm.

\section{ACKNOWLEDGMENT}

The authors would like to thank Doct. Martina Panfili and Eng. Antonio Ornatelli and all the other members of the Network Control Laboratory for the fruitful discussions and preliminary simulations.

\section{REFERENCES}

[1] F. Adamsky, M. Aubigny, F. Battisti, M. Carli, F. Cimorelli, T. Cruz, A. D. Giorgio, C. Foglietta, A. Galli, A. Giuseppi, F. Liberati, A. Neri, S. Panzieri, F. Pascucci, J. Proenca, P. Pucci, L. Rosa, and R. Soua, "Integrated protection of industrial control systems from cyber-attacks: the ATENA approach," International Journal of Critical Infrastructure Protection, vol. 21, pp. 72-82, June 2018.

[2] M. Faisal, M. A. Hannan, P. J. Ker, A. Hussain, M. B. Mansor, and F. Blaabjerg, "Review of energy storage system technologies in microgrid applications: Issues and challenges," Ieee Access, vol. 6, pp. 35143-35164, 2018.

[3] L. Tronchin, M. Manfren, and B. Nastasi, "Energy efficiency, demand side management and energy storage technologies-a critical analysis of possible paths of integration in the built environment," Renewable and Sustainable Energy Reviews, vol. 95, pp. 341-353, 2018.

[4] F. Liberati, A. D. Giorgio, A. Giuseppi, A. Pietrabissa, and F. Delli Priscoli, "Efficient and risk-aware control of electricity distribution grids," IEEE Systems Journal, pp. 1-12, 2020.

[5] A. Fiaschetti, V. Suraci, and F. Delli Priscoli, "The SHIELD framework: How to control security, privacy and dependability in complex systems," in 2012 Complexity in Engineering (COMPENG). Proceedings, IEEE, June 2012.

[6] Y. M. Atwa and E. El-Saadany, "Optimal allocation of ess in distribution systems with a high penetration of wind energy," IEEE Transactions on Power Systems, vol. 25, no. 4, pp. 1815-1822, 2010.
[7] A. D. Giorgio, F. Liberati, R. Germana, M. Presciuttini, L. R. Celsi, and F. Delli Priscoli, "On the control of energy storage systems for electric vehicles fast charging in service areas," in 2016 24th Mediterranean Conference on Control and Automation (MED), IEEE, June 2016.

[8] J. Feltes and C. Grande-Moran, "Black start studies for system restoration," in 2008 IEEE Power and Energy Society General Meeting Conversion and Delivery of Electrical Energy in the 21st Century, IEEE, July 2008.

[9] P. Demetriou, M. Asprou, and E. Kyriakides, "A real-time controlled islanding and restoration scheme based on estimated states," IEEE Transactions on Power Systems, vol. 34, pp. 606-615, Jan. 2019.

[10] B. Chen, C. Chen, J. Wang, and K. L. Butler-Purry, "Sequential service restoration for unbalanced distribution systems and microgrids," IEEE Transactions on Power Systems, vol. 33, pp. 1507-1520, Mar. 2018.

[11] Y. Zhao, Z. Lin, Y. Ding, Y. Liu, L. Sun, and Y. Yan, "A model predictive control based generator start-up optimization strategy for restoration with microgrids as black-start resources," IEEE Transactions on Power Systems, vol. 33, pp. 7189-7203, Nov. 2018.

[12] M. Dietmannsberger, X. Wang, F. Blaabjerg, and D. Schulz, "Restoration of low-voltage distribution systems with inverter-interfaced DG units," IEEE Transactions on Industry Applications, vol. 54, pp. 53775386, Sept. 2018.

[13] G. Andersson, P. Donalek, R. Farmer, N. Hatziargyriou, I. Kamwa, P. Kundur, N. Martins, J. Paserba, P. Pourbeik, J. Sanchez-Gasca, R. Schulz, A. Stankovic, C. Taylor, and V. Vittal, "Causes of the 2003 major grid blackouts in north america and europe, and recommended means to improve system dynamic performance," IEEE Transactions on Power Systems, vol. 20, pp. 1922-1928, Nov. 2005.

[14] A. D. Giorgio, A. Giuseppi, F. Liberati, and A. Pietrabissa, "Controlled electricity distribution network black start with energy storage system support," in 2017 25th Mediterranean Conference on Control and Automation (MED), IEEE, July 2017.

[15] C. Warren, "Overview of 1366-2001 the full use guide on electric power distribution reliability indices," in IEEE Power Engineering Society Summer Meeting, IEEE, 2005.

[16] R. A. Jabr, "Radial distribution load flow using conic programming," IEEE Tran. on Power Systems, vol. 21, pp. 1458-1459, Aug 2006.

[17] H. Ahmadi and J. R. Martí, "Mathematical representation of radiality constraint in distribution system reconfiguration problem," International Journal of Electrical Power \& Energy Systems, vol. 64, pp. 293299, Jan. 2015.

[18] P. Kundur, N. J. Balu, and M. G. Lauby, Power system stability and control, vol. 7. McGraw-hill New York, 1994.

[19] R. A. Jabr, R. Singh, and B. C. Pal, "Minimum loss network reconfiguration using mixed-integer convex programming," IEEE Transactions on Power Systems, vol. 27, pp. 1106-1115, May 2012.

[20] J. Pereira, J. Alves, and M. Matos, "Optimization of electrical distribution network operation based on EPSO," in 2015 18th International Conference on Intelligent System Application to Power Systems (ISAP), IEEE, Sept. 2015.

[21] J. Bezanson, A. Edelman, S. Karpinski, and V. B. Shah, "Julia: A fresh approach to numerical computing," SIAM review, vol. 59, no. 1, pp. 65-98, 2017.

[22] I. Dunning, J. Huchette, and M. Lubin, "Jump: A modeling language for mathematical optimization," SIAM Review, vol. 59, no. 2, pp. 295320, 2017.

[23] L. Gurobi Optimization, "Gurobi optimizer reference manual," 2019. 